\title{
Role of Advective Inertia in Active Nematic Turbulence
}

\author{
Colin-Marius Koch(1) and Michael Wilczek ${ }^{*}{ }^{*}$ \\ Max Planck Institute for Dynamics and Self-Organization, Am Faßberg 17, 37077 Göttingen, Germany \\ and Faculty of Physics, Georg-August-Universität Göttingen, Friedrich-Hund-Platz, 1, 37077 Göttingen, Germany
}

(Received 18 February 2021; accepted 22 October 2021; published 23 December 2021)

\begin{abstract}
Suspensions of active agents with nematic interactions exhibit complex spatiotemporal dynamics such as mesoscale turbulence. Since the Reynolds number of microscopic flows is very small on the scale of individual agents, inertial effects are typically excluded in continuum theories of active nematic turbulence. Whether active stresses can collectively excite inertial flows is currently unclear. To address this question, we investigate a two-dimensional continuum theory for active nematic turbulence. In particular, we compare mesoscale turbulence with and without the effects of advective inertia. We find that inertial effects can influence the flow already close to the onset of the turbulent state and, moreover, give rise to large-scale fluid motion for strong active driving. A detailed analysis of the kinetic energy budget reveals an energy transfer to large scales mediated by inertial advection. While this transfer is small in comparison to energy injection and dissipation, its effects accumulate over time. The inclusion of friction, which is typically present in experiments, can compensate for this effect. The findings suggest that the inclusion of inertia and friction may be necessary for dynamically consistent theories of active nematic turbulence.
\end{abstract}

DOI: 10.1103/PhysRevLett.127.268005

Active matter on the microscale consists of motile agents, such as bacteria [1-4] and cells [5,6], filaments driven by motor proteins [7-11], motile algae [12-14], or colloids $[15,16]$. Suspended densely in a liquid, they form so-called active fluids, in which the flow is driven on the scale of the agents [17]. Their collective behavior can lead to complex mesoscale phenomena, such as active turbulence, which is reminiscent of driven hydrodynamic flows and has been observed, e.g., in suspensions of bacteria $[1,18,19]$ and in microtubule kinesin mixtures $[11,20]$. The latter case is an example of an active liquid crystal, for which continuum models have been adapted from liquid crystal theory to include active stresses that excite the flow field [21-23].

An individual microscopic agent is subject to drag forces in the fluid, which are large compared to its inertial forces due to its small size, mass, and propulsion speed [24-26]. As a consequence, the agent's dynamics are dominated by its self-propulsion and the viscous damping of the fluid. However, the collective motion that leads to active turbulence has been found to significantly exceed velocities found for individual agents [1,27]. This raises the question of whether the collective behavior of many active agents, in

Published by the American Physical Society under the terms of the Creative Commons Attribution 4.0 International license. Further distribution of this work must maintain attribution to the author(s) and the published article's title, journal citation, and DOI. Open access publication funded by the Max Planck Society. principle, can excite flows in which inertial effects become apparent.

Here, we address this question with a detailed study on the impact of inertia on dense suspensions of active agents in the framework of an established two-dimensional continuum model of active nematic liquid crystals [28-31], which has been related to experimental results [32]. In this model for wet active matter, hydrodynamic interactions have either been taken into account using Stokes flow [23,33,34], or unsteady Stokes flow [31,35-37]. Here, we additionally include the full Navier-Stokes dynamics to test whether active stresses can excite collective inertial flows. In particular, we explore under which conditions large-scale flow patterns like the ones observed in two-dimensional hydrodynamic turbulence can emerge [38-40]. To this end, we perform numerical simulations of this model with and without inertial advection and friction and compare various flow statistics in the different regimes.

To study the impact of advective inertia on the fluid flow of two-dimensional active nematic turbulence, we use the continuum equations established for a dense suspension of motile, aligning particles [37], which originate from the well-studied Beris-Edwards model of liquid crystal theory [33,41-43]. In essence, the equations couple the fluid flow to an order parameter field describing the nematic order. The fluid flow is described by the incompressible $(\nabla \cdot \boldsymbol{u}=0)$ Navier-Stokes equation, which in nondimensional form reads

$$
\begin{aligned}
\operatorname{Re}_{n}\left(\partial_{t} \boldsymbol{u}+\boldsymbol{u} \cdot \nabla \boldsymbol{u}\right)= & -\nabla p+\Delta \boldsymbol{u}-R_{f} \boldsymbol{u} \\
& +\frac{1}{\mathrm{Er}} \nabla \cdot\left[\boldsymbol{\sigma}_{e}-R_{a} \boldsymbol{\sigma}_{a}\right]
\end{aligned}
$$


In this nondimensional form based on the nematic scales $m_{n}, l_{n}$, and $t_{n}$ (cf. Supplemental Material [44]), the equation weights the inertial forces with the microscopic Reynolds number $\operatorname{Re}_{n}=\rho u_{n} l_{n} / \eta$, and the elastic and active stresses with the inverse of the Ericksen number $\mathrm{Er}=\eta u_{n} l_{n} / K$, where $\rho$ is the mass density, $\eta$ the dynamic viscosity, and $K$ an elastic constant. In contrast to classical fluids, active nematics feature an additional elastic stress $\boldsymbol{\sigma}_{e}=-\lambda S \Delta \boldsymbol{Q}+(\Delta \boldsymbol{Q}) \boldsymbol{Q}-\boldsymbol{Q}(\Delta \boldsymbol{Q})+\lambda S \boldsymbol{Q}\left(S^{2}-1\right)$ [51] as well as the active stress $\sigma_{a}=Q$, which couple the orientational field $\boldsymbol{Q}$ to the flow field. While the elastic stress describes the reaction of the flow to the particles' reorientation, the active stress models the impact of motility on the fluid flow [21]. The ratio $R_{a}=l_{n}^{2} / l_{a}^{2}$ of nematic and active length scales varies the relative strength between the stresses. It depends on the nematic $l_{n}^{2}=K / C$ and the active $l_{a}^{2}=K / \alpha$ length scales defined based on the material constants $K$ and $C$ and the activity $\alpha$. In addition to the original model [37], we include linear friction as a simple approximation to interactions between a two-dimensional active nematic layer and its surrounding. We control its influence with the nondimensional friction number $R_{f}=l_{n}^{2} / l_{f}^{2}$, which we define via the friction length scale $l_{f}^{2}=\eta / \mu$ based on the friction coefficient $\mu$. The orientational order is described by the symmetric and traceless second-rank tensor $Q_{i j}=S\left(n_{i} n_{j}-\delta_{i j} / 2\right)$, where $\boldsymbol{n}$ is a director and $S=\sqrt{2 \operatorname{Tr}\left(\boldsymbol{Q}^{2}\right)}$ quantifies the local nematic order. The orientational field evolves in its nondimensional form according to

$$
\partial_{t} \boldsymbol{Q}+\boldsymbol{u} \cdot \nabla \boldsymbol{Q}=\lambda \boldsymbol{S E}-\boldsymbol{W} \boldsymbol{Q}+\boldsymbol{Q} \boldsymbol{W}+\Delta \boldsymbol{Q}-\boldsymbol{Q}\left(S^{2}-1\right),
$$

where $E_{i j}=\left(\partial_{i} u_{j}+\partial_{j} u_{i}\right) / 2$ and $W_{i j}=\left(\partial_{i} u_{j}-\partial_{j} u_{i}\right) / 2$ are the symmetric and antisymmetric parts of the velocity gradient, respectively. The alignment parameter $\lambda$ controls the particles' reaction to shear [33]. The system's relaxation to a uniformly aligned state is effectively described by the diffusion of boundaries via $\Delta \boldsymbol{Q}$ as well as local alignment via $\boldsymbol{Q}\left(S^{2}-1\right)$.

As a measure for the impact of inertial effects on the fluid flow, we focus on the self-advection term $\boldsymbol{u} \cdot \nabla \boldsymbol{u}$ in Eq. (1) which has not been considered in previous work [31,35-37] due to the low-Reynolds-number approximation. For direct comparison, we performed numerical simulations on a periodic domain of size $L$. We use a pseudospectral scheme for spatial discretization with a fourth-order Runge-Kutta scheme for integration in time. We scanned a range of values for the active number $R_{a}$ which includes the onset of active nematic turbulence and ranges well into the turbulent regime (cf. Supplemental Material [44], Table S1). Within the studied parameter regime, we practically define the onset of active nematic turbulence by determining the lowest active number showing the creation and annihilation of defects $\left(R_{a} \approx 0.05\right.$, cf. Fig. 3(a) and Supplemental Material [44], Fig. S1). As a starting point, we fix the microscopic Reynolds and the Ericksen number $\operatorname{Re}_{n}=\mathrm{Er}=0.1$ as in [37] (cf. Supplemental Material [44], Table S2). In order to study the statistics independent of the initial conditions, we equilibrated the system until it reached a statistically stationary state before analyzing the data (cf. Supplemental Material [44]). To achieve well-converged statistics, we averaged over an ensemble of $N_{\text {ens }}=100$ realizations with independent random initial conditions as well as over the simulated time in the statistically stationary state.

Active stresses in this model for active nematic turbulence are able to induce inertial effects. A visualization of the main observations is given in Fig. 1. For that, we computed an ensemble with a fixed set of parameters $\left(R_{a}=0.2\right)$. This ensemble transitions over time from the original $(\mathrm{O})$, over the inertial (I) regime, to the regime with inertia and friction (IF), by numerically switching on advection $(\boldsymbol{u} \cdot \nabla \boldsymbol{u})$ at the beginning of the first, and linear friction $\left(R_{f}>0\right)$ at the beginning of the second transition. Changes from one to the next regime are clearly visible in

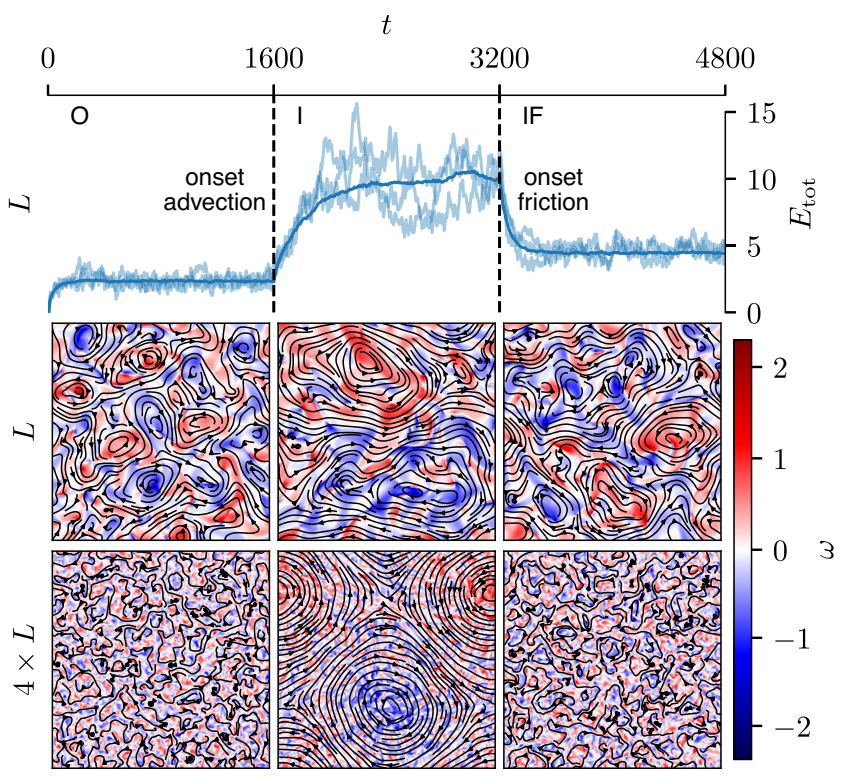

FIG. 1. Advective inertia causes the formation of large-scale flow patterns and an increase in kinetic energy. Linear friction reduces these effects. Top: time evolution of the total kinetic energy $E_{\text {tot }}$ in the original $(\mathrm{O})$, inertial (I), and inertial with friction (IF) regimes. Displayed are individual realizations (light blue) and the ensemble average (dark blue) with indicated times at which advection and friction are switched on (dashed lines). Center and bottom: Snapshots of the vorticity $\omega=\partial_{x} u_{y}-\partial_{y} u_{x}$ (color map) and the velocity field (contour lines) taken from the statistically stationary states in each respective regime (box sizes $L$ and $4 L, L=204.8, \quad R_{a}=0.2, \quad \operatorname{Re}_{n}=0.1, \quad \mathrm{Er}=0.1$, $R_{f}=7.5 \times 10^{-4}$, see also Supplemental Movies S1-S3). 
the visualization as well as in the kinetic energy of the flow field.

Visually, the flow field in the original regime exhibits complex motion displayed by counterrotating vortices (red and blue patches) which are interspersed by small vortex dipoles. The dipoles originate from the active stresses produced by topological defects in the orientational field and move through the system. While these basic features persist in the inertial regime, the scale of motion increases drastically: rotating patterns on the scale of half the box size emerge. These patterns fluctuate strongly, decay, and reform over time (cf. Supplemental Material [44], movies $\mathrm{S} 1-\mathrm{S} 3$ ). If the simulation domain is large enough (compare results for $L$ and $4 L$ ), they form metastable condensatelike patterns. The formation of homogeneous condensates, i.e., large homogeneous vortices as known from classical twodimensional turbulence $[39,52,53]$ and other continuum models of active turbulence [54,55], seems to be prevented by the small, propagating vortex dipoles. However, the observations suggest that for a sufficiently large system, the disturbances by individual defects become small in comparison to the large-scale flow pattern, and a clearer and more stable condensatelike structure forms.

The kinetic energy in the original regime fluctuates around a mean value. At the onset of advection, however, the energy increases until it saturates to a higher mean value. While the initial increase in energy is visually accompanied by a build-up of more chaotic and longerranged motion, the new steady state corresponds to the fully developed flow with large-scale motion.

The appearance of large-scale motion together with the increase in kinetic energy demonstrate that the inclusion of advective inertia changes the dynamics and statistics of the fluid flow drastically. Furthermore, this suggests a connection between the scales of the flow and the increase in kinetic energy.

Indeed, a spectral analysis of the kinetic energy, i.e., its spectrum and budget, explains the observations (Fig. 2). The kinetic energy spectrum provides a scale-by-scale characterization of the kinetic energy and is defined as [40]:

$$
E(k, t)=\frac{1}{2 \Delta k} \sum_{k \leq|\boldsymbol{k}|<k+\Delta k}|\hat{\boldsymbol{u}}(\boldsymbol{k}, t)|^{2},
$$

where $\Delta k=2 \pi / L$. Comparing the original and inertial regime [Fig. 2(a)], the spectrum displays a prominent rise in energy at small wave numbers, i.e., large scales. In contrast, energy increases only a little at higher wave numbers corresponding to smaller scales. Consequently, the increase in total kinetic energy in the inertial regime primarily stems from energy accumulating at large scales. This fits well with the observation of dominant large-scale motion. The spectral energy budget allows us to study scale by scale how much energy each term in Eq. (1) injects into or dissipates from the flow:

$$
\partial_{t} E=T+D+S_{e}+S_{a}+F,
$$

where $T(k, t)$ is the inertial energy transfer due to advection, $D(k, t)$ the viscous dissipation, $S_{e}(k, t)$ the elastic dissipation, $S_{a}(k, t)$ the active injection, and $F(k, t)$ the dissipation through friction (cf. Supplemental Material [44]). Interestingly, energy injection by active stresses occurs on a broad range of scales, featuring a maximum at intermediate scales [Fig. 2(b)]. In the steady state of the original regime, viscous and elastic forces dissipate the injected energy, i.e., the three contributions balance each other at each scale [56]. In the inertial regime however, the advection term mediates an energy transfer between scales [Fig. 2(c)]. It extracts energy around the scale of maximal injection and transfers the majority towards larger and a small portion towards smaller scales. This is quantified by the energy flux [Fig. 2(d)]

$$
\Pi(k, t)=\int_{k}^{\infty} d k^{\prime} T\left(k^{\prime}, t\right),
$$

where a negative flux indicates an inverse transfer to larger and a positive flux a direct transfer to smaller scales. This flux of energy between scales leads to an overall increase in energy, which is compensated for on small scales by
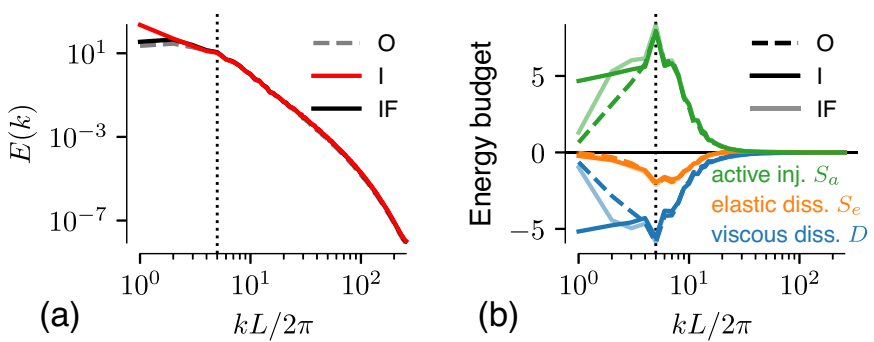
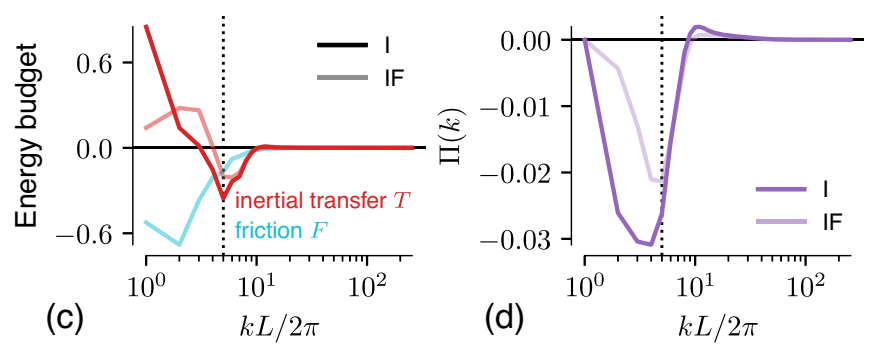

FIG. 2. Advective inertia mediates an inverse energy transfer to the largest scales leading to an accumulation of energy and large-scale motion. Linear friction can compensate for this by directly dissipating the transferred energy. The original (O) regime is compared to the inertial (I) regime as well as to the regime with inertia and friction (IF): ensemble- and time-averaged (a) kinetic energy spectrum $E(k)$, (b) and (c) energy budget contributions (4), (d) energy flux $\Pi(k)$. The dotted lines indicate the scale with maximal active energy injection. ( $L=204.8, R_{a}=0.2, \operatorname{Re}_{n}=0.1, \mathrm{Er}=0.1, R_{f}=7.5 \times 10^{-4}$ ) 

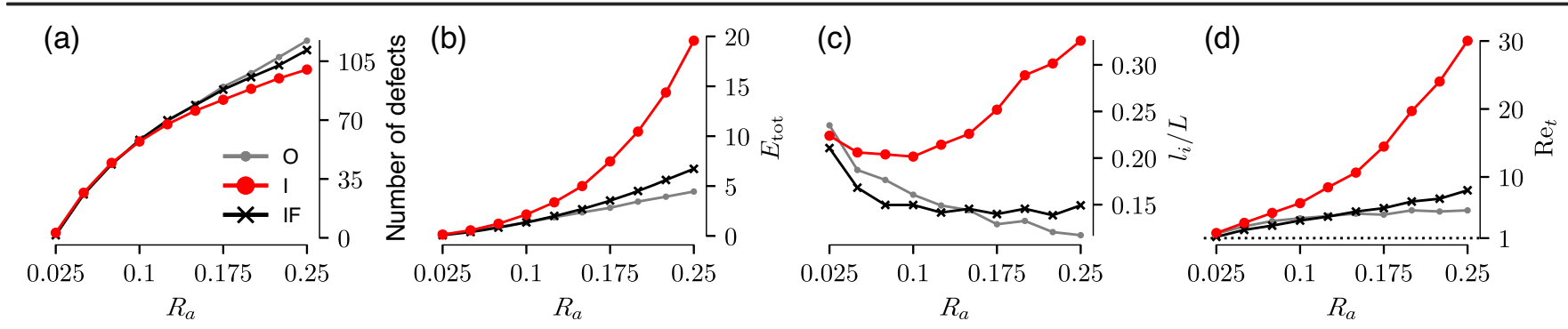

FIG. 3. Advective inertia impacts statistics of the fluid flow already for weak and increasingly for stronger active forcing. The original (O) regime is compared to the inertial (I) regime as well as to the regime with inertia and friction (IF): ensemble- and time-averaged (a) number of defects, (b) total kinetic energy $E_{\text {tot }}$, (c) integral length $l_{i}$, (d) turbulent Reynolds number Re $e_{t}$ computed from (b) and (c). $\left(L=204.8, \operatorname{Re}_{n}=0.1, \mathrm{Er}=0.1, R_{f}=7.5 \times 10^{-4}\right)$

viscous dissipation but remains initially unbalanced on large scales. Only after an initial accumulation of energy on large scales, viscous dissipation becomes strong enough to compensate for any further flux mediated by advective inertia. Interestingly, the active stress contribution results in an even larger injection on these scales when the large-scale energy is increased, which is balanced by a similar increase in viscous dissipation [Fig. 2(b)]. Remarkably, the peak magnitude of the energy transfer due to advection is about one order of magnitude smaller than the peak magnitudes of the active stress contribution and viscous dissipation, indicating that inertial advection has a comparably small effect at any particular instant, which, however, builds up over time.

The spectral analysis demonstrates that advective inertia is responsible for an inverse transfer of energy towards larger scales, at which energy accumulates, resulting in the observed large-scale motion. To quantify how the strength of active forcing mediates the inertial effects, we vary $R_{a}$ ranging from the onset of active nematic turbulence until deep into the turbulent regime (Fig. 3).

Recall that we define the onset of active nematic turbulence by where defects first spontaneously form and annihilate $\left[R_{a} \approx 0.05\right.$, cf. Fig. 3(a) and Supplemental Material [44], Fig. S1]. The number of defects [57] increases with activity in both regimes as can be expected for increased disorder due to stronger flow. Interestingly, it is smaller in the inertial than in the original regime.

As indicated in Fig. 1, the total kinetic energy $E_{\text {tot }}(t)=$ $\sum_{k} E(k, t) \Delta k$ is higher in the inertial than in the original regime. As can be expected for stronger active driving, the difference grows larger with activity [Fig. 3(b)]. Interestingly, it is already nonzero at the onset of active nematic turbulence and grows continuously. This suggests that inertial effects are non-negligible for all activities studied in this parameter regime.

The turbulent Reynolds number based on the integral scale is a typical measure for the importance of inertial effects in comparison to viscous dissipation in turbulent flows: $\operatorname{Re}_{t}=\sqrt{E_{\mathrm{tot}}} l_{i} / \nu$, where $\nu=\eta / \rho$ is the kinematic viscosity and $l_{i}$ is the integral length scale (cf. Supplemental Material [44]). Indeed, in both regimes, the emerging turbulent Reynolds number is larger than unity for all activities [Fig. 3(d)], which means that inertial effects are not negligible (cf. Supplemental Material [44]). The difference in Reynolds numbers between the original and the inertial regime increases, as the kinetic energy, with activity. This is readily understood because the turbulent Reynolds number is proportional to the root of the kinetic energy, and because the integral length scale increases with the emergence of large-scale patterns in the inertial regime [Fig. 3(c)].

The parameter scan for the activity demonstrates that advective inertia changes the fluid flow increasingly with activity, already starting at the onset of active nematic turbulence. Consistent modeling, therefore, requires the inclusion of the advection term in this parameter regime. Furthermore, surface friction has been found to influence quasi-two-dimensional active nematic layers experimentally $[20,32]$ as well as numerically $[58,59]$. This motivates the addition of linear friction to the model (regime with inertia and friction in Fig. 1). Linear friction dissipates energy primarily on large scales, which contain the most energy [Fig. 2(c)]. It thereby counteracts the accumulation of energy through the inverse transfer and prohibits the formation of large-scale flow [Figs. 1 and 2(a)]. While it can restore the system to a state very similar to the original regime (Fig. 3), i.e., without advection and friction, its impact on the flow depends on the relative magnitude of friction coefficient and activity. A scan of the friction coefficient shows that the qualitative flow features and statistics transition smoothly from the inertial to the original regime and beyond with increasing friction number (Supplemental Material [44], Figs. S3 and S4).

So far, we have varied the active forcing strength at fixed microscopic Reynolds and Ericksen numbers. To get a broader overview, Fig. 4 shows the turbulent Reynolds number as a function of the microscopic Reynolds number and the Ericksen number for two different activities for the case including inertia and friction. For small microscopic Reynolds numbers and comparably large Ericksen numbers, the turbulent Reynolds number is generally small, consistent with recent findings [45] (cf. Supplemental Material [44], Table S2, Fig. S5). However, as the 

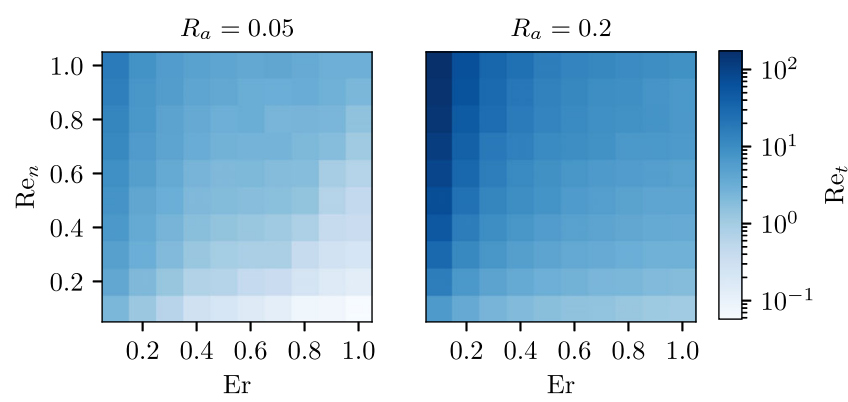

FIG. 4. The importance of inertia, quantified by the turbulent Reynolds number $\mathrm{Re}_{t}$, increases directly with the microscopic Reynolds number $\operatorname{Re}_{n}$ and inversely with the Ericksen number. The simulations were performed in the regime with inertia and friction and were averaged over 30 independent random initial conditions. $\left(L=204.8, R_{f}=7.5 \times 10^{-4}\right)$

Ericksen number is decreased, the turbulent Reynolds number increases. The largest turbulent Reynolds numbers emerge for the smallest Ericksen numbers, where they can have values significantly larger than one even if the microscopic Reynolds number is small. In this regime, active stresses can effectively drive the flow, thereby exciting inertial effects.

To summarize, by comparing a continuum model for active nematic turbulence with and without inertial advection, we find that inertial effects can significantly alter the fluid flow by an accumulation of kinetic energy on the largest scales of the flow. In particular, we observe largescale motion in the form of condensatelike flow patterns when inertial effects are present. Remarkably, inertial effects start to play a role already for small activities, provided the active stresses can effectively drive the flow.

Linear friction, included to model surface friction present in the experimental setups, compensates for the effects of inertial advection by dissipating the inversely transferred energy, resulting effectively in a flow similar to the system without advection and friction. Including inertial advection and friction appears therefore necessary for a consistent theoretical description of active nematic turbulence in certain parameter ranges. Based on this work, a careful assessment of experimental parameters could enable predictions about the significance of inertial effects and their interplay with surface friction, which we hope will spur future experimental investigations in that direction.

We thank the reviewers for helpful comments. This work was supported by the Max Planck Society. M. W. gratefully acknowledges a Fulbright-Cottrell Award grant.

*michael.wilczek@ds.mpg.de

[1] C. Dombrowski, L. Cisneros, S. Chatkaew, R. E. Goldstein, and J. O. Kessler, Phys. Rev. Lett. 93, 098103 (2004).

[2] A. Sokolov, I. S. Aranson, J. O. Kessler, and R.E. Goldstein, Phys. Rev. Lett. 98, 158102 (2007).
[3] E. Lushi, H. Wioland, and R. E. Goldstein, Proc. Natl. Acad. Sci. U.S.A. 111, 9733 (2014).

[4] F. Peruani, J. Starruß, V. Jakovljevic, L. Søgaard-Andersen, A. Deutsch, and M. Bär, Phys. Rev. Lett. 108, 098102 (2012).

[5] R. Kemkemer, V. Teichgräber, S. Schrank-Kaufmann, D. Kaufmann, and H. Gruler, Eur. Phys. J. E 3, 101 (2000).

[6] G. Duclos, C. Erlenkämper, J.-F. Joanny, and P. Silberzan, Nat. Phys. 13, 58 (2017).

[7] F. Nédélec, T. Surrey, A. C. Maggs, and S. Leibler, Nature (London) 389, 305 (1997).

[8] T. Surrey, F. Nédélec, S. Leibler, and E. Karsenti, Science 292, 1167 (2001).

[9] V. Schaller, C. Weber, C. Semmrich, E. Frey, and A. R. Bausch, Nature (London) 467, 73 (2010).

[10] Y. Sumino, K. H. Nagai, Y. Shitaka, D. Tanaka, K. Yoshikawa, H. Chaté, and K. Oiwa, Nature (London) 483, 448 (2012).

[11] T. Sanchez, D. T. Chen, S. J. DeCamp, M. Heymann, and Z. Dogic, Nature (London) 491, 431 (2012).

[12] K. Drescher, R. E. Goldstein, N. Michel, M. Polin, and I. Tuval, Phys. Rev. Lett. 105, 168101 (2010).

[13] K. Drescher, J. Dunkel, L. H. Cisneros, S. Ganguly, and R. E. Goldstein, Proc. Natl. Acad. Sci. U.S.A. 108, 10940 (2011).

[14] F. von Rüling, F. Kolley, and A. Eremin, Colloid Polym. Sci. 299, 289 (2021).

[15] A. Bricard, J.-B. Caussin, N. Desreumaux, O. Dauchot, and D. Bartolo, Nature (London) 503, 95 (2013).

[16] X. Cao, E. Panizon, A. Vanossi, N. Manini, and C. Bechinger, Nat. Phys. 15, 776 (2019).

[17] D. Saintillan, Annu. Rev. Fluid Mech. 50, 563 (2018).

[18] H. H. Wensink, J. Dunkel, S. Heidenreich, K. Drescher, R. E. Goldstein, H. Löwen, and J. M. Yeomans, Proc. Natl. Acad. Sci. U.S.A. 109, 14308 (2012).

[19] J. Dunkel, S. Heidenreich, K. Drescher, H. H. Wensink, M. Bär, and R. E. Goldstein, Phys. Rev. Lett. 110, 228102 (2013).

[20] P. Guillamat, J. Ignés-Mullol, S. Shankar, M. C. Marchetti, and F. Sagués, Phys. Rev. E 94, 060602(R) (2016).

[21] R. A. Simha and S. Ramaswamy, Phys. Rev. Lett. 89, 058101 (2002).

[22] S. Thampi and J. Yeomans, Eur. Phys. J. Special Topics 225, 651 (2016).

[23] A. Doostmohammadi, J. Ignés-Mullol, J. M. Yeomans, and F. Sagués, Nat. Commun. 9, 3246 (2018).

[24] E. M. Purcell, Am. J. Phys. 45, 3 (1977).

[25] E. Lauga and T. R. Powers, Rep. Prog. Phys. 72, 096601 (2009).

[26] J. Elgeti, R. G. Winkler, and G. Gompper, Rep. Prog. Phys. 78, 056601 (2015).

[27] N. H. Mendelson, A. Bourque, K. Wilkening, K. R. Anderson, and J. C. Watkins, J. Bacteriol. 181, 600 (1999).

[28] D. Marenduzzo, E. Orlandini, and J. M. Yeomans, Phys. Rev. Lett. 98, 118102 (2007).

[29] S. P. Thampi, R. Golestanian, and J. M. Yeomans, Phys. Rev. Lett. 111, 118101 (2013).

[30] M. C. Marchetti, J.-F. Joanny, S. Ramaswamy, T. B. Liverpool, J. Prost, M. Rao, and R. A. Simha, Rev. Mod. Phys. 85, 1143 (2013).

[31] L. Giomi, L. Mahadevan, B. Chakraborty, and M. F. Hagan, Phys. Rev. Lett. 106, 218101 (2011). 
[32] P. Guillamat, J. Ignés-Mullol, and F. Sagués, Proc. Natl. Acad. Sci. U.S.A. 113, 5498 (2016)

[33] E. J. Hemingway, P. Mishra, M. C. Marchetti, and S. M. Fielding, Soft Matter 12, 7943 (2016).

[34] R. Alert, J.-F. Joanny, and J. Casademunt, Nat. Phys. 16, 682 (2020).

[35] L. Giomi, L. Mahadevan, B. Chakraborty, and M. F. Hagan, Nonlinearity 25, 2245 (2012).

[36] L. Giomi, M. J. Bowick, P. Mishra, R. Sknepnek, and M. C. Marchetti, Phil. Trans. R. Soc. A 372, 20130365 (2014).

[37] L. Giomi, Phys. Rev. X 5, 031003 (2015).

[38] G. Boffetta and S. Musacchio, Phys. Rev. E 82, 016307 (2010).

[39] G. Boffetta and R. E. Ecke, Annu. Rev. Fluid Mech. 44, 427 (2012).

[40] A. Alexakis and L. Biferale, Phys. Rep. 767-769, 1 (2018).

[41] A. N. Beris and B. J. Edwards, Thermodynamics of Flowing Systems: With Internal Microstructure, (Oxford University Press, New York, 1994), p. 535-549.

[42] T. Qian and P. Sheng, Phys. Rev. E 58, 7475 (1998).

[43] H. Abels, G. Dolzmann, Y. Liu, Adv. Differ. Equ. 21, 109 (2016).

[44] See Supplemental Material at http://link.aps.org/supplemental/ 10.1103/PhysRevLett.127.268005 for more details. The Supplemental Material includes Refs. [29,33,37,45-50].

[45] L. Carenza, L. Biferale, and G. Gonnella, Europhys. Lett. 132, 44003 (2020).

[46] D. Tritton, Physical Fluid Dynamics (Oxford Science Publication, Clarendon Press, Oxford, 1998).
[47] S. B. Pope, Turbulent Flows (Cambridge University Press, Cambridge, England, 2000).

[48] P. A. Davidson, Turbulence: An Introduction for Scientists and Engineers (Oxford University Press, New York, 2015).

[49] J. Urzay, A. Doostmohammadi, and J. M. Yeomans, J. Fluid Mech. 822, 762 (2017).

[50] G. Boffetta, A. Cenedese, S. Espa, and S. Musacchio, Europhys. Lett. 71, 590 (2005).

[51] In this work, we treat $\lambda S$ as varying in space and time [with $\lambda=$ const, $S(\boldsymbol{x}, t)$ ] as was done in Ref. [37]. Since $\lambda S=$ const is used in other works [29,33], we compared the investigated statistics in both cases and found qualitatively identical results.

[52] L. M. Smith and V. Yakhot, Phys. Rev. Lett. 71, 352 (1993).

[53] L. M. Smith and V. Yakhot, J. Fluid Mech. 274, 115 (1994).

[54] M. Linkmann, G. Boffetta, M. C. Marchetti, and B. Eckhardt, Phys. Rev. Lett. 122, 214503 (2019).

[55] M. Linkmann, M. C. Marchetti, G. Boffetta, and B. Eckhardt, Phys. Rev. E 101, 022609 (2020).

[56] The elastic stress also injects energy at small scales, which becomes more visible in the enstrophy budget. [cf. Supplemental Material Fig. S2 [44] and [45]].

[57] D. Huterer and T. Vachaspati, Phys. Rev. D 72, 043004 (2005).

[58] S. P. Thampi, R. Golestanian, and J. M. Yeomans, Phys. Rev. E 90, 062307 (2014).

[59] K. Thijssen, L. Metselaar, J. M. Yeomans, and A. Doostmohammadi, Soft Matter 16, 2065 (2020). 